\title{
Climatic factors associated with amyotrophic lateral sclerosis: a spatial analysis from Taiwan
}

\author{
Ching-Piao Tsai ${ }^{1 *}$, Charles Tzu-Chi Lee ${ }^{2 *}$ \\ ${ }^{1}$ Neurology, Neurological Institute, Taipei Veterans General Hospital and National Yang-Ming University, \\ Taipei, Taiwan; ${ }^{2}$ Department of Public Health, Kaohsiung Medical University, Kaohsiung, Taiwan
}

\begin{abstract}
Few studies have assessed the spatial association of amyotrophic lateral sclerosis (ALS) incidence in the world. The aim of this study was to identify the association of climatic factors and ALS incidence in Taiwan. A total of 1,434 subjects with the primary diagnosis of ALS between years 1997 and 2008 were identified in the national health insurance research database. The diagnosis was also verified by the national health insurance programme, which had issued and providing them with "serious disabling disease (SDD) certificates". Local indicators of spatial association were employed to investigate spatial clustering of agestandardised incidence ratios in the townships of the study area. Spatial regression was utilised to reveal any association of annual average climatic factors and ALS incidence for the 12-year study period. The climatic factors included the annual average time of sunlight exposure, average temperature, maximum temperature, minimum temperature, atmospheric pressure, rainfall, relative humidity and wind speed with spatial autocorrelation controlled. Significant correlations were only found for exposure to sunlight and rainfall and it was similar in both genders. The annual average of the former was found to be negatively correlated with ALS, while the latter was positively correlated with ALS incidence. While accepting that ALS is most probably multifactorial, it was concluded that sunlight deprivation and/or rainfall are associated to some degree with ALS incidence in Taiwan.
\end{abstract}

Keywords: amyotrophic lateral sclerosis, sunlight exposure, rainfall, climate, Taiwan.

\section{Introduction}

Amyotrophic lateral sclerosis (ALS) is a fatal neurodegenerative syndrome with no known cause, except for a small proportion of cases which are genetically inherited. The development of the disease is likely to involve both genetic and environmental risk factors (Caller et al., 2012). According to previous research in the United States of America (USA) and Europe, some studies reported that the incidence of ALS showed a north to south latitude gradient, i.e. the risk of disease was found to be higher at higher (northerly) latitudes (Annegers et al., 1991). Another study from southern Italy showing a similar gradient supports this finding but does not otherwise explain the small, seemingly uncorrelated variations of ALS incidence in Europe (Logroscino et al., 2005). Furthermore, later work by the same first author concluded that incidence of this disease is homogeneous across Europe (Logroscino et al., 2010). Apart from the findings reported above,

Corresponding author:

Charles Tzu-Chi Lee

Department of Public Health

Kaohsiung Medical University

No. 100, Shihcyuan $1^{\text {st }}$ Rd, Kaohsiung City 80708, Taiwan (R.O.C.)

Tel. + 8867 312-1101; Fax +886 7 311-0811

E-mail: charles@kmu.edu.tw

*The authors have contributed equally to this article research on the spatial distribution features of ALS incidence is scarce. The analysis of geographical patterns such as pollution (Caller et al., 2009; CaparrosLefebvre et al., 2006) and sunlight-deprivation (Sato et al., 1997) provide possible clues for the cause of ALS but no hard facts. Some studies employed spatial analysis techniques to examine spatial clusters of ALS but they only attempted to identify the geographical disparities in its distribution and did not offer any clear association with environmental factors (Caller et al., 2012).

The Republic of China (R.O.C.) - Taiwan is an island, $390 \mathrm{~km}$ long and $140 \mathrm{~km}$ wide, located in the northwestern part of the Pacific Ocean. It can be divided into three major geologic provinces: the Central Range, the Western Foothills and the Coastal Range. The Central Range forms the backbone ridge of the island, where the older continental shelf and slope sediments are raised to a maximum elevation of almost $4,000 \mathrm{~m}$ (Liu et al., 2009). The population is $98 \%$ Han Chinese and $2 \%$ indigenous aboriginal peoples. The varieties in geographical characteristics, combined with a relatively uniform ethnic composition, provide an optimal opportunity for examining the association between natural environment exposure and ALS incidence. In this study, spatial analysis was utilised with population-based data and geographical data to investigate the association between climatic factors and ALS incidence. The results present suggestions for further research into identifying the effect of natural environmental factors on ALS incidence. 


\section{Material and methods}

\section{Population data}

Taiwan consists of 358 townships and the total population at the census of the year 2000 was 21.3 million. The township populations ranged from 1,472 to 521,689. The average size of the townships was 100.9 $\mathrm{km}^{2}$, ranging from 1.0 to $1,621.2 \mathrm{~km}^{2}$. In this study, the patient township was defined as their workplace to better account for outdoor exposure to the climatic factors. Each township was treated as an individual unit in the analysis.

\section{National health insurance in Taiwan}

The national health insurance (NHI) programme, a government-run, single-payer insurance system was established in Taiwan in 1995. When the enrolment of NHI was made mandatory in December 2008, there were 22.918 million individual members nationwide corresponding to a coverage rate of $99.5 \%$. The NHI bureau requires registration of all cases of serious disabling diseases (SDDs) such as chronic renal failure, myasthenia gravis, cancer and ALS without which SDD certification cannot be granted. The final approval of the SDD certificates requires strict evaluation by the Department of Health in Taiwan. Patients with SDD certificates are eligible for exemption from insurance premiums and co-payments.

By December 2008, there were 37,099 registered medical doctors and 553 registered neurology specialists in Taiwan, while there were 790,621 individuals with SDD certificates, which constituted $3.4 \%$ of the total population.

\section{Sample}

This study was a population-based, retrospective cohort investigation that analysed information from the national health insurance research database (NHIRD), which includes data on outpatients, ambulatory patients, inpatients, dental services and drug prescriptions. ALS cases are identified by code 335.20 of the International Classification of Disease, Ninth Revision (ICD-9).

The diagnosis of ALS is based on the El Escorial criteria (Brooks, 1994) by the clinical neurologist seeing the patient, and medical records are sent to the NHI bureau. Another group of neurologists there verify the medical records and confirm the diagnosis according to the El Escorial criteria (Brooks, 1994). All ALS par- ticipating patients fulfilled the El Escorial criteria. In addition, only ALS patients with SDD certification were included in the study. All cases were verified by linking encrypted identification numbers with the SDD certificates. There were 1,434 ALS cases included in this study, which covered a period of 12 years (1997-2008). The data for the analysis were provided by the NHIRD without personal identification.

\section{Statistical associations investigated}

In order to investigate the association between the township ALS incidence and the climatic factors, records of the annual average of sunlight exposure hours, average temperatures, maximum temperatures, minimum temperatures, atmospheric pressure, rainfall, relative humidity and wind speed for the years 1997 and 2008 were retrieved from 23 major observation stations maintained by the Central Weather Bureau (Fig. 1a). The ambient, climatic factors of each of the 358 townships were represented by records from its nearest observation station.

Cigarette smoking has been proposed as a risk factor for ALS (Wang et al., 2011). Although the association of smoking with ALS risk is mild (Alonso et al., 2010), we included the smoking prevalence in the explanatory adjusted model for comparison purposes. Data on the prevalence of cigarette smoking in the 358 townships were retrieved from a survey conducted in 2002 by the Department of Health.

\section{Statistical analysis}

We first included the 1,434 ALS cases from 1997 to 2008 based on the NHIRD and SDD registry. Secondly, the indirect age- and gender-standardised incidence rate (ASIR) of ALS based on the year 2000 census population of township was calculated. The expected numbers of ALS in each township was calculated by the weighted average of age-specific ALS incidence in Taiwan and the age distributions of the township populations. Thus, the expected numbers in the townships with variant population and age distribution were taken into account. The ASIR values were then calculated as the ratio of observed $v s$. the expected numbers of ALS cases for the 358 townships. An ASIR value of 1 indicates that the township incidence equals the average incidence of Taiwan as a whole. Similarly, an ASIR value $>1$ illustrates that the township incidence is larger than the average national incidence, while an ASIR $<1$ indicates that the incidence is less than the average incidence. In 

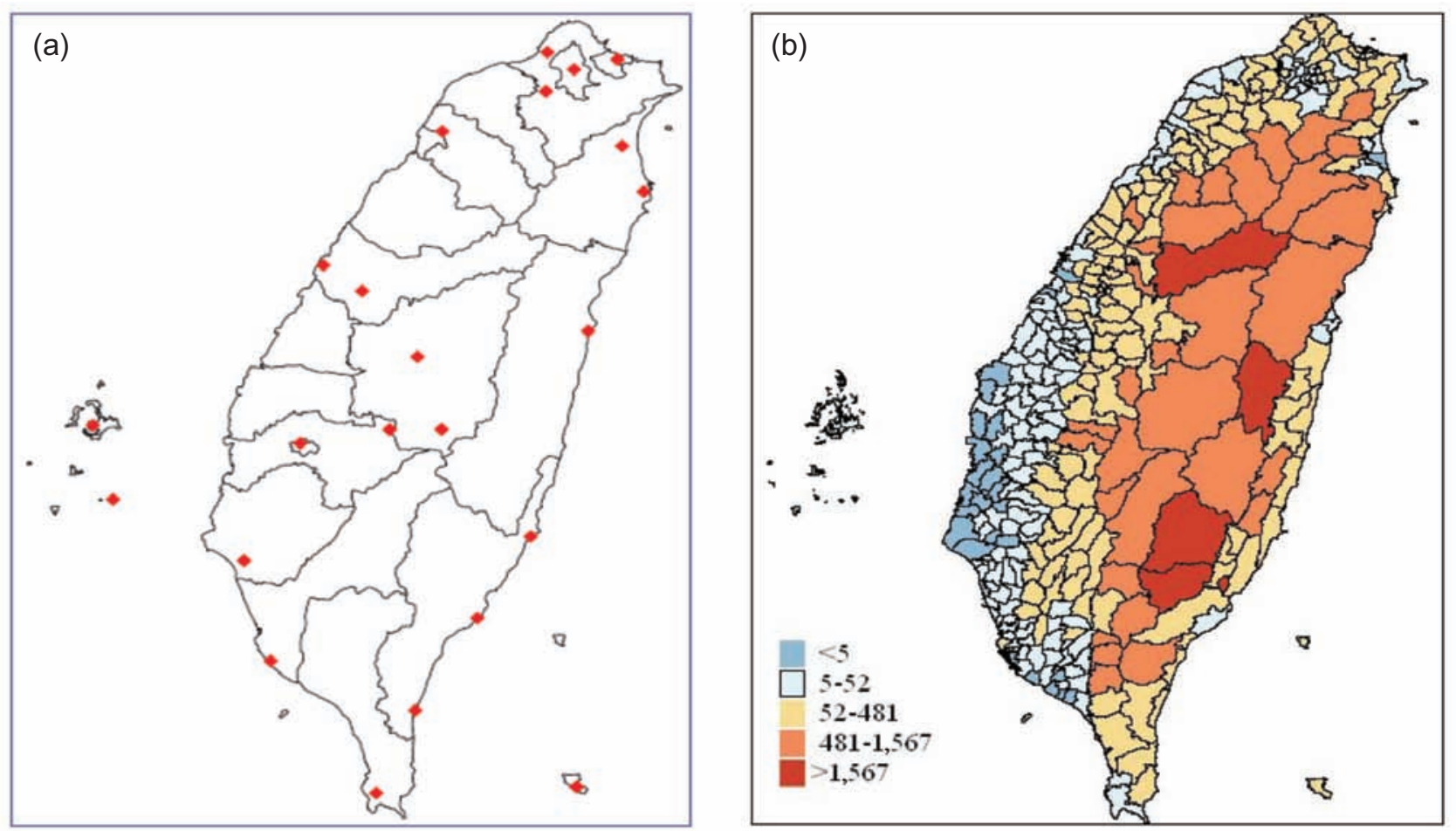

Fig. 1. Weather station locations (a) and township altitudes ( $m$ above the mean sea level) in Taiwan (b).

this study, the data were all analysed with the known population gender and age distributions within the townships investigated.

The local indicator of spatial association was employed in order to measure the spatial cluster of the township ASIR values (Anselin, 1995). The local indicator of spatial association is widely used in the spatial epidemiology in order to demonstrate clusters of incidence or other health indicators. Spatial regression (Medade and Earickson, 2000) was used to reveal the effect of climatic factors on ALS incidence with the spatial autocorrelation being controlled. To analyse the spatial data, the correlations between regions were taken into account in order to provide valid, scientific evidence.

The townships were divided into urban and rural categories and included in the model for control purposes. The unadjusted model, explanatory adjusted and the finally adjusted model with stepwise selection were shown by the spatial autoregressive model. The unadjusted model had the explanatory variables added to the regression model one at a time to reveal pair-wise association between these variables and ALS incidence without controlling for potential confounding factors with the exception of spatial autocorrelation. The finally adjusted model used stepwise selection to find the most significant explanatory variables for ALS incidence. This selection process starts with the unadjusted models to find the most significant unadjusted explanatory variable and other variables are added one at a time. Each step allows backward elimination to make it possible to decide if the latest added variable needed to be removed after controlling the variables added previously. The explanatory adjusted model indicated that smoking and urbanization should be included without selection for controlling purposes and that stepwise selection should be chosen for the other climatic factors.

The spatial autoregressive model (Anselin, 1988), also called the spatial lag model, takes on the following form:

$$
y=\rho W y+X \beta+\varepsilon
$$

where $y$ is a vector of observations on the dependent variable, $W y$ a spatially lagged dependent variable for the weights matrix $W, X$ a matrix of observations on the explanatory variables, $\varepsilon$ the vector of error terms following $\mathrm{N}\left(0, \sigma^{2}\right)$, while $\rho$ and $\beta$ are parameters. The $\mathrm{P}$-value for significance was set at 0.05 . The model was tested first with all cases included and then with subgroups consisting of the two genders. GeoDa095.i (http://geodacenter.asu.edu/) was used to estimate spatial autoregressive models and plot the map. 


\section{Results}

\section{ALS case characteristics}

The ALS patients were predominately between 45 and 64 years of age and included 894 males and 540 females. The male-to-female ratio was thus 1.66 . With respect to residency, most patients $(1,120$ or $78.1 \%)$ were from urban townships. We used the health insurance premium groups as proxy for economic status of the patients, $60 \%$ of the cases were found to be either receiving social-welfare support or had a dependent member of the family (Table 1 ).

Table 1. Characteristics of individuals diagnosed with ALS in Taiwan for the period 1997-2008.

\begin{tabular}{lcc}
\hline Characteristic & No. & $\%$ \\
\hline Gender & & \\
Female & 540 & 37.7 \\
Male & 894 & 62.3 \\
Age (years) & & \\
1 1-24 & 71 & 5.0 \\
$25-44$ & 248 & 17.3 \\
$45-64$ & 691 & 48.2 \\
$\geq 65$ & 424 & 29.6 \\
Residence & & \\
Rural & 314 & 21.9 \\
Urban & 1,120 & 78.1 \\
Insurance income & & \\
Fixed premium and dependent & 859 & 59.9 \\
Less than 20,000 New Taiwan Dollars (NTD) & 315 & 22.0 \\
20,000 New Taiwan Dollarsb or more & 260 & 18.1 \\
\hline
\end{tabular}

${ }^{\mathrm{a}}$ Total number $=1,434 ;{ }^{\mathrm{b}} 1$ US\$ $=32.1$ NTD in 2008.

\section{Climatic factors}

The climatic variables emanated from the 23 major observation stations in Taiwan (Table 2). The average temperature during the 12-year study period was $21.7^{\circ} \mathrm{C}$ (standard deviation $(\mathrm{SD})=4.8^{\circ} \mathrm{C}$ ). Because of Taiwan's diverse geography with many elevations of different heights (Fig. 1b), the average temperature ranged from $4.2{ }^{\circ} \mathrm{C}$ to $25.1{ }^{\circ} \mathrm{C}$ at these stations. The average annual rainfall was 2,392 $\mathrm{mm}(\mathrm{SD}=974 \mathrm{~mm})$, ranging from 1,013 to $4,440 \mathrm{~mm}$. The annual average sunlight exposure was 1,769 hours ranging from 1,277 to 2,234 hours of sunlight. From the eight climatic factors studied, the coefficient of variation (CV) showed that the highest was the wind speed at 60.3 and the lowest was the relative humidity at 5.1. The $\mathrm{CV}$ is defined as the ratio of SD to the mean. Since CV is a term without unit, it can be used to compare the variation between variables with different units. The CVs of rainfall and sunlight exposure hours were 40.8 and 18.1 , respectively.

\section{The spatial cluster of ALS incidence}

As can be seen the overview of spatial clusters in Taiwan (Fig. 2), ALS was found to be significantly more common in northern Taiwan than in the other geographical regions. The townships depicted in red in the figure indicate significant clusters with particularly high ASIR values ("hot spots"), while the blue colour indicates "cold spots", which are characterised by low ASIR values. The light red and light blue colours in the figure indicate outliers, the former indicating townships with high ASIR values surrounded by neighbours with extremely low ASIR values and the latter township with low ASIR values surrounded

Table 2. Climate statistics from 23 main observation stations in Taiwan for the period 1997-2008.

\begin{tabular}{|c|c|c|c|c|c|}
\hline Climatic factors & Average & $\mathrm{SD}^{\mathrm{a}}$ & $\mathrm{CV}^{\mathrm{b}}(\%)$ & Minimum & Maximum \\
\hline Temperature $\left({ }^{\circ} \mathrm{C}\right)$ & 21.7 & 4.8 & 22.1 & 4.2 & 25.1 \\
\hline Maximum temperature $\left({ }^{\circ} \mathrm{C}\right)$ & 25.4 & 4.5 & 17.6 & 9.4 & 28.8 \\
\hline Minimum temperature $\left({ }^{\circ} \mathrm{C}\right)$ & 19.0 & 5.0 & 26.4 & 0.8 & 22.5 \\
\hline Atmospheric pressure (100 pa) & 974.5 & 91.4 & 9.4 & 642.2 & $1,013.1$ \\
\hline Rainfall (mm) & 2,392 & 974 & 40.8 & 1,013 & 4,440 \\
\hline Relative humidity (\%) & 79.3 & 4.0 & 5.1 & 74.1 & 88.9 \\
\hline Sunshine exposure (100 hours) & 1,769 & 32.9 & 18.1 & 1,277 & 2,234 \\
\hline Wind speed $(\mathrm{m} / \mathrm{sec})$ & 3.2 & 1.9 & 60.3 & 1.1 & 8.1 \\
\hline
\end{tabular}

${ }^{a}$ Standard deviation; ${ }^{\mathrm{b}}$ coefficient of variation. 

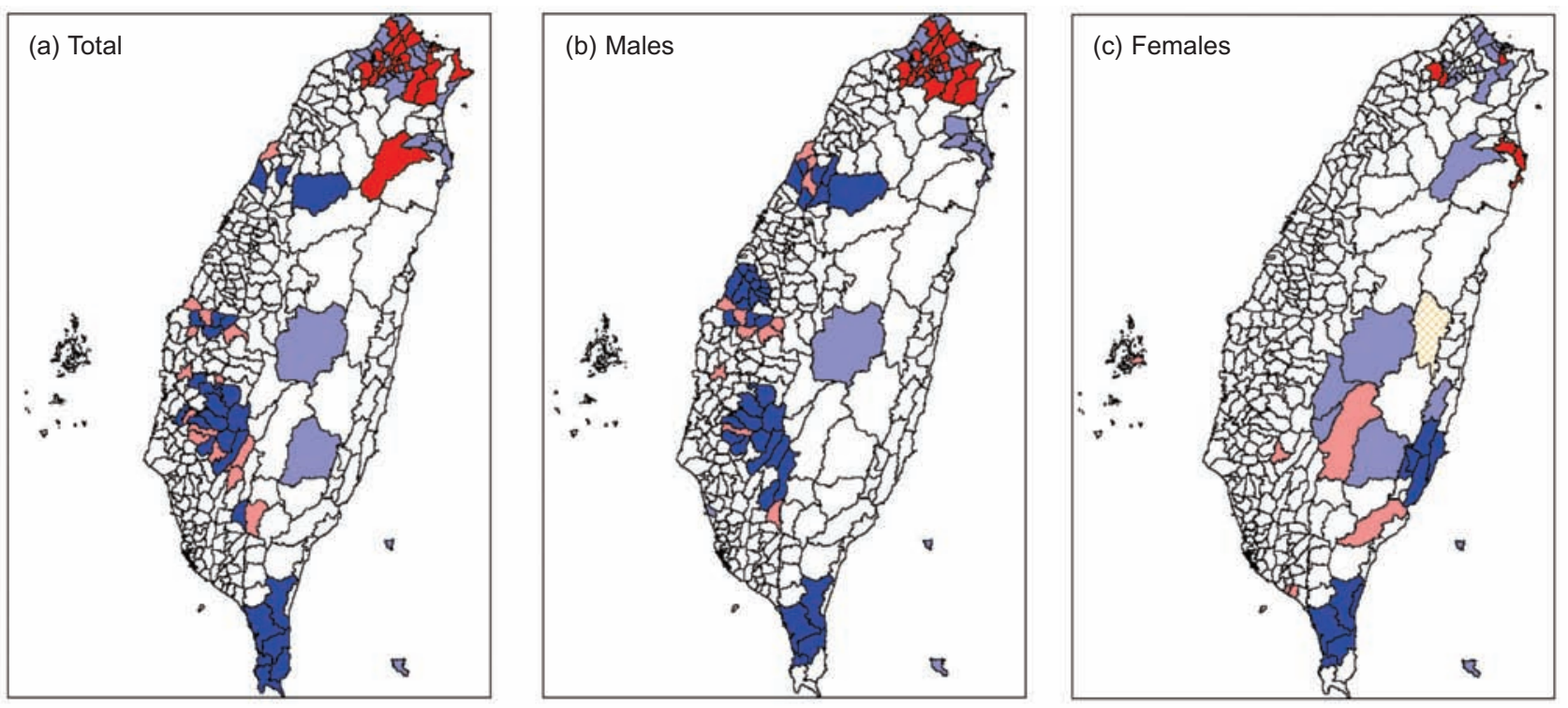

Hot spots Cold spots Local high-neighbours low $\quad$ Local low-neighbours high $\square$ Not significant

Fig. 2. Local spatial clusters of ALS age-standardised incidence ratio in Taiwan for the period 1997-2008.

by neighbours with extremely high ASIR values. Townships in white indicate areas without significant local clusters $(P>0.05)$. It was discovered that male cases dominated in the hot spots in the north of Taiwan (Figs. 2a, b and c).

The association between climatic factors and ALS incidence

Of the eight climatic factors under investigation, the spatial distribution of ALS incidence yielded a similar geographical distribution to rainfall, i.e. the values were found to be higher in the Northeast and the central, highly mountain areas, while the sunlight exposure demonstrated higher value in the southwest areas (Fig. 3). The spatial autoregressive model indicated that the rainfall and the extent of sunlight exposure were independent predictors of the total ALS incidence. It was also noted from the study that every additional $100 \mathrm{~mm}$ rise in annual rainfall effected an increase of the township ASIR value by 0.028 ( $\mathrm{P}<0.001)$. Conversely, the final adjusted model showed that with every 100 hours rise in annual sunlight exposure resulted in a decrease of the township ASIR value by 0.068 (P $<0.001$ ) in the final adjusted model (Table 3 ). In the gender subgroup analysis, the results for the eight cli-
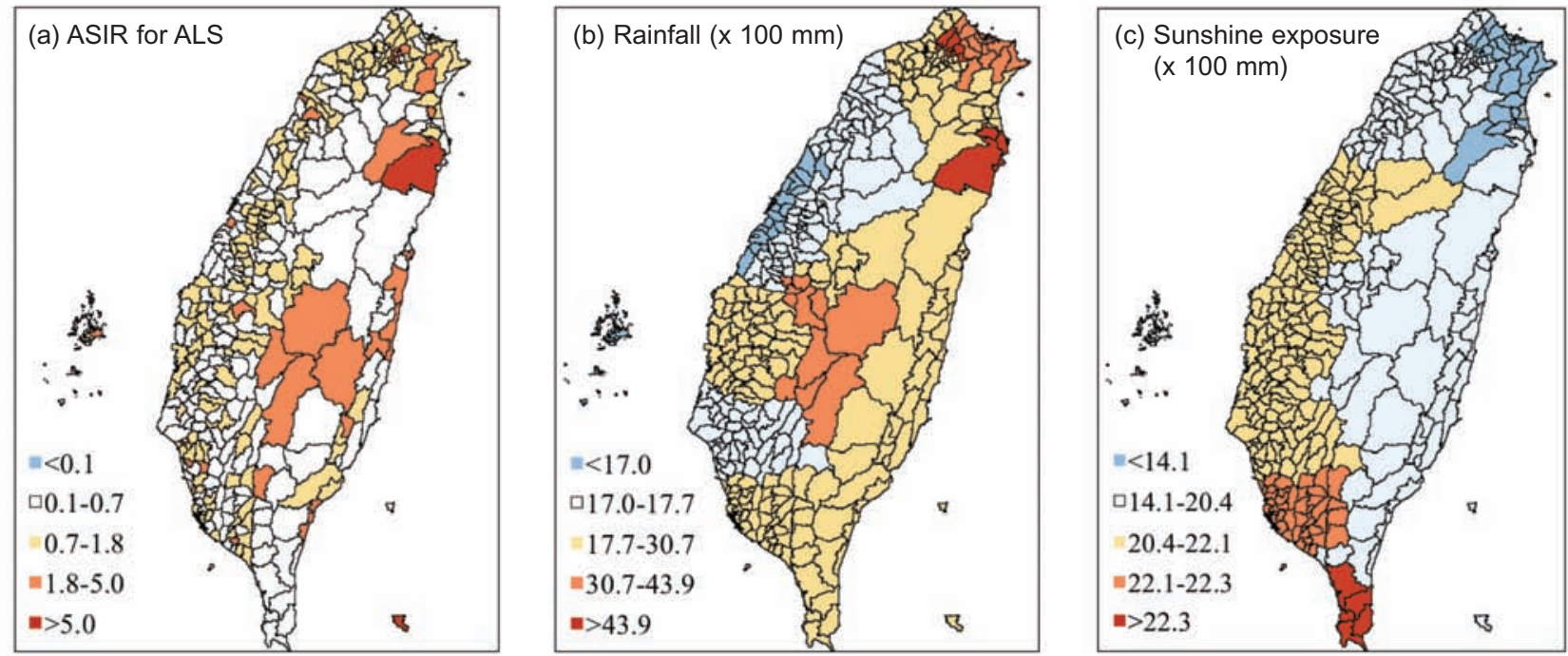

Fig. 3. Age- and gender-standardised incidence ratios (ASIR), rainfall and sun exposure in Taiwan for the period 1997-2008. 
Table 3. Spatial autoregressive model on age- and gender-standardised ALS incidence ratios in Taiwan 1997-2008.

\begin{tabular}{|c|c|c|c|c|c|c|}
\hline \multirow{2}{*}{ Climatic factors (annual average) } & \multicolumn{2}{|c|}{ Unadjusted } & \multicolumn{2}{|c|}{ Explanatory adjusted } & \multicolumn{2}{|c|}{ Finally adjusted } \\
\hline & Estimate & P-value & Estimate & P-value & Estimate & P-value \\
\hline \multicolumn{7}{|l|}{ (a) Whole sample } \\
\hline Temperature $\left({ }^{\circ} \mathrm{C}\right)$ & -0.0220 & 0.036 & & & & \\
\hline Maximum temperature $\left({ }^{\circ} \mathrm{C}\right)$ & -0.0299 & 0.006 & & & & \\
\hline Minimum temperature $\left({ }^{\circ} \mathrm{C}\right)$ & -0.0164 & 0.111 & & & & \\
\hline Atmospheric pressure (100 pa) & -0.0010 & 0.077 & & & & \\
\hline Rainfall $(100 \mathrm{~mm})$ & 0.0398 & $<0.001$ & 0.0295 & $<0.001$ & 0.0282 & $<0.001$ \\
\hline Relative humidity (\%) & 0.0610 & $<0.001$ & & & & \\
\hline Sunshine exposure (100 hours) & -0.1027 & $<0.001$ & -0.0641 & $<0.001$ & -0.0678 & $<0.001$ \\
\hline Wind speed $(\mathrm{m} / \mathrm{sec})$ & 0.0208 & 0.513 & & & & \\
\hline Smoking prevalence $(\%)$ & 0.0006 & 0.911 & 0.0057 & 0.267 & & \\
\hline Urbanization (rural vs. urban) & -0.3171 & $<0.001$ & -0.3545 & $<0.001$ & -0.3349 & $<0.001$ \\
\hline \multicolumn{7}{|l|}{ (b) Male cases } \\
\hline Temperature $\left({ }^{\circ} \mathrm{C}\right)$ & -0.0140 & 0.284 & & & & \\
\hline Maximum temperature $\left({ }^{\circ} \mathrm{C}\right)$ & -0.0240 & 0.074 & & & & \\
\hline Minimum temperature $\left({ }^{\circ} \mathrm{C}\right)$ & -0.0068 & 0.598 & & & & \\
\hline Atmospheric pressure (100 pa) & -0.0005 & 0.438 & & & & \\
\hline Rainfall $(100 \mathrm{~mm})$ & 0.0405 & $<0.001$ & 0.0251 & $<0.001$ & 0.0286 & $<0.001$ \\
\hline Relative humidity (\%) & 0.0667 & $<0.001$ & & & & \\
\hline Sunshine exposure (100 hours) & -0.1000 & $<0.001$ & -0.0722 & 0.002 & -0.0692 & 0.001 \\
\hline Wind speed $(\mathrm{m} / \mathrm{sec})$ & 0.0745 & 0.057 & & & & \\
\hline Smoking prevalence $(\%)$ & 0.0039 & 0.563 & -0.0005 & 0.947 & & \\
\hline Urbanization (rural vs. urban) & -0.3863 & $<0.001$ & -0.4222 & $<0.001$ & -0.4236 & $<0.001$ \\
\hline \multicolumn{7}{|l|}{ (c) Female cases } \\
\hline Temperature $\left({ }^{\circ} \mathrm{C}\right)$ & -0.0415 & 0.008 & & & & \\
\hline Maximum temperature $\left({ }^{\circ} \mathrm{C}\right)$ & -0.0474 & 0.003 & & & & \\
\hline Minimum temperature $\left({ }^{\circ} \mathrm{C}\right)$ & -0.0377 & 0.014 & & & & \\
\hline Atmospheric pressure (100 pa) & -0.0018 & 0.025 & & & & \\
\hline Rainfall $(100 \mathrm{~mm})$ & 0.0384 & 0.001 & 0.0328 & 0.001 & 0.0296 & 0.003 \\
\hline Relative humidity (\%) & 0.0536 & 0.005 & & & & \\
\hline Sunshine exposure (100 hours) & -0.0828 & 0.001 & -0.0209 & 0.040 & -0.0337 & 0.017 \\
\hline Wind speed $(\mathrm{m} / \mathrm{sec})$ & -0.0900 & 0.056 & & & & \\
\hline Smoking prevalence $(\%)$ & 0.0079 & 0.334 & 0.0155 & 0.062 & & \\
\hline Urbanization (rural $v s$. urban) & -0.2466 & 0.018 & -0.2136 & 0.023 & -0.2121 & 0.013 \\
\hline
\end{tabular}

Controlled by spatial autocorrelation.

matic factors under investigation were similar to that of the total. The association between sunshine-exposure and ALS incidence was similar in both males and females. We also found this to be true of rainfall (Table 3). In this study, the cigarette smoking prevalence of townships did not have a significant effect on the total ALS incidence and the explanatory adjusted analysis controlling cigarette smoking prevalence did not change our findings.

\section{Discussion}

The ambient climatic factors are highly different between the various regions, e.g. the western and southern Taiwan, which are situated behind the Central Range, are characterised by north-eastern trade winds and reduced rainfall leading to more sunny days in the year. Northern Taiwan, on the other hand, sees the north-eastern trade winds leaving the area with less sunlight exposure in autumn and winter. Eastern Taiwan, constituted by rift valleys extending in the longitudinal direction, receives less rainfall compared to the North.

Because the association between climatic factors and ALS incidence is still unclear, we used one of model selection methods, the stepwise procedure, to find the most significant factors associated with ALS incidence in the exploratory study. Meanwhile, we also provided the explanatory adjusted model, which included cigarette smoking prevalence and urbanization. The results of these two approaches strategies were similar. After controlling for the spatial autocorrelation and urbanization in the final adjusted model, sunlight-deprivation and/or rainfall appeared to be the most significant independent predictors. This finding conflicts with the preliminary, individual-level study by Steiner et al. (1994). One possible explanation might be that results from this study were based on limited data, 
whereas our study was based on a larger population.

It should be acknowledged that the limited number of stations keeping long-term, climatic records in Taiwan might be a limitation (Fig. 1a) by diluting the effect of climatic factors on ALS incidence in small regions leading to underestimation. Despite the fact that this was a 12-year population-based study, only 1,434 ALS cases were verified, reflecting the fact that we studied a rare disease in a small country. It would be beneficial if other countries, perhaps with higher ALS incidence, could replicate a similar analysis for confirmation. In addition, disease onset and diagnosis may differ according to the economic status and residence of patients, as these variables could affect access to neurologists. It was unfortunate that the date of ALS onset was not available in the database. Finally, migration can also produce a bias because of misclassification of residences. In this ecological study, we did not have any information about whether and when people in moving within or between townships. Indeed, that was one of the reasons that patient township was defined as their workplace that was better known.

A previous, ecological study suggests that the incidence of ALS is homogeneous across Europe (Logroscino et al., 2005). However, that study used larger units than ours that was based on the township. Importantly, our study population was also highly homogeneous (98\% Han Chinese) and located in an island country. Since ALS is possibly affected both by genetic and environmental factors, it might be easier to find the important climatic factors by investigating a highly homogeneous population and focus the analysis on small areas. Besides, natural environment exposure must, by definition, focus on populations rather than individuals, which make to approach more difficult when dealing with a relatively uncommon disease. The strength of our design is that we were able to study a relatively large, representative and homogeneous sample, which provides a higher level of statistical power. The principal limitation with any study of this nature is that causality is difficult to infer and we can only say that findings are consistent with a given hypothesis (Chen et al., 2012).

If the positive association between sunlight-deprivation and ALS incidence is indeed causal, it could be linked to the hypovitaminosis D in ALS patients reported by Sato et al. (1997). These authors found that the serum concentration of 25-hydroxyvitamin D was significantly lower in ALS patients than in controls. ALS is a progressive neurodegenerative disease, in which misfolding and aggregation of proteins, e.g. superoxide dismutase 1 (SOD1), are central features, while vitamin $\mathrm{D}$ has antioxidant, anti-inflammatory and neuroprotective properties and may mitigate this pathology. Interestingly, a recent study has demonstrated that vitamin $\mathrm{D}$ has a crucial role in activating human T cells (von Essen et al., 2010). Thus, it is rational to infer that vitamin $\mathrm{D}$ supplement has the potential to accelerate clearance of misfolded SOD1 aggregates through inducing autophagy (Shen, 2011). Even if the pathogenesis of ALS is multifactorial, vitamin $\mathrm{D}$ is safe and may delay progression of ALS by acting on several aspects of the disease (Karam and Scelsa, 2011). Although our findings indicate sunlightdeprivation as a possible factor, further research is required to strengthen this hypothesis by studying the role of vitamin D in the development of ALS.

A higher number of children born in late summer and early winter in Australia develop ALS later in life, a trend which decreases between mid-winter and early summer (Pamphlett and Fang, 2012). Similar patterns have been seen in Japan (Ajdacic-Gross et al., 1998) and Sweden (Fang et al., 2009). Monthly average humidity correlates positively with the numbers of births that lead to ALS later in life in Australia, Sweden and Japan. Thus, early life factors related to weather conditions such as increased humidity could have an association with ALS and this needs to be further investigated (Pamphlett and Fang, 2012). In our study, both rainfall and humidity were associated with ALS incidence in the unadjusted models. Although the association between humidity and ALS incidence disappeared in the final adjusted model, when controlled by rainfall, the association between rainfall and ALS incidence can still be associated with humidity effect in some way, perhaps because humidity tends to lead to more infectious diseases and allergies. However, not only humidity at birth but also life time needs to be further investigated in relation to the incidence of ALS.

\section{Acknowledgements}

This study was based on the data from the national health insurance research database provided by the Bureau of National Health Insurance, Department of Health, Taiwan and was managed by the National Health Research Institutes. The interpretations and conclusions contained herein are our own and do not represent those of the Bureau of National Health Insurance, Department of Health, or of the National Health Research Institutes. This research was supported by Ching-Ling Foundation of Taipei Veterans General Hospital. The foundation had no role in the study design, data collection and analysis, decision to publish, or preparation of the manuscript. 


\section{References}

Ajdacic-Gross V, Wang J, Gutzwiller F, 1998. Season of birth in amyotrophic lateral sclerosis. Eur J Epidemiol 14, 359-361.

Alonso A, Logroscino G, Hernan MA, 2010. Smoking and the risk of amyotrophic lateral sclerosis: a systematic review and meta-analysis. J Neurol Neurosurg Psychiatry 81, 1249-1252.

Annegers JF, Appel S, Lee JR, Perkins P, 1991. Incidence and prevalence of amyotrophic lateral sclerosis in Harris county, Texas, 1985-1988. Arch Neurol 48, 589-593.

Anselin L, 1988. Spatial econometrics: methods and models. Dordrecht: Kluwer Academic Publishers.

Anselin L, 1995. Local indicators of spatial association. Geograph Anal 27, 94-115.

Brooks BR, 1994. El Escorial World Federation of Neurology criteria for the diagnosis of amyotrophic lateral sclerosis. Subcommittee on Motor Neuron Diseases/Amyotrophic Lateral Sclerosis of the World Federation of Neurology Research Group on Neuromuscular Diseases and the El Escorial "Clinical limits of amyotrophic lateral sclerosis" workshop contributors. J Neurol Sci 124, 96-107.

Caller TA, Doolin JW, Haney JF, Murby AJ, West KG, Farrar HE, Ball A, Harris BT, Stommel EW, 2009. A cluster of amyotrophic lateral sclerosis in New Hampshire: a possible role for toxic cyanobacteria blooms. Amyotroph Lateral Scler 10, 101-108.

Caller TA, Field NC, Chipman JW, Shi X, Harris BT, Stommel EW, 2012. Spatial clustering of amyotrophic lateral sclerosis and the potential role of BMAA. Amyotroph Lateral Scler 13, 25-32.

Caparros-Lefebvre D, Steele J, Kotake Y, Ohta S, 2006. Geographic isolates of atypical Parkinsonism and tauopathy in the tropics: possible synergy of neurotoxins. Mov Disord 21, 1769-1771.

Chen VC, Stewart R, Lee CT, 2012. Weekly lottery sales volume and suicide numbers: a time series analysis on national data from Taiwan. Soc Psychiatry Psychiatr Epidemiol 47, 1055 1059.

Fang F, Valdimarsdottir U, Bellocco R, Ronnevi LO, Sparen P,
Fall K, Ye W, 2009. Amyotrophic lateral sclerosis in Sweden, 1991-2005. Arch Neurol 66, 515-519.

GeoDa Center for Geospatial Analysis and Computation. Available at: http://geodacenter.asu.edu/ (accessed on October 2012).

Karam C, Scelsa SN, 2011. Can vitamin D delay the progression of ALS? Med Hypotheses 76, 643-645.

Liu C, Linde AT, Sacks IS, 2009. Slow earthquakes triggered by typhoons. Nature 459, 833-836.

Logroscino G, Beghi E, Zoccolella S, Palagano R, Fraddosio A, Simone IL, Lamberti P, Lepore V, Serlenga L, 2005. Incidence of amyotrophic lateral sclerosis in southern Italy: a population based study. J Neurol Neurosurg Psychiatry 76, 1094-1098.

Logroscino G, Traynor BJ, Hardiman O, Chio A, Mitchell D, Swingler RJ, Millul A, Benn E, Beghi E, EURALS, 2010. Incidence of amyotrophic lateral sclerosis in Europe. J Neurol Neurosurg Psychiatry 81, 385-390.

Medade MS, Earickson RJ, 2000. Medical geography (2 ed.). New York: Guilford.

Pamphlett R, Fang F, 2012. Season and weather patterns at time of birth in amyotrophic lateral sclerosis. Amyotroph Lateral Scler 13, 459-464.

Sato Y, Honda Y, Asoh T, Kikuyama M, Oizumi K, 1997. Hypovitaminosis D and decreased bone mineral density in amyotrophic lateral sclerosis. Eur Neurol 37, 225-229.

Shen L, 2011. Further support for vitamin D supplement in delaying the progression of ALS. Med Hypotheses 77, 698.

Steiner I, Birmanns B, Panet A,1994. Sun exposure and amyotrophic lateral sclerosis. Ann Intern Med 120, 893.

von Essen MR, Kongsbak M, Schjerling P, Olgaard K, Odum N, Geisler C, 2010. Vitamin D controls T cell antigen receptor signaling and activation of human T cells. Nat Immunol 11, 344-349.

Wang H, O’Reilly EJ, Weisskopf MG, Logroscino G, McCullough ML, Thun MJ, Schatzkin A, Kolonel LN, Ascherio A, 2011. Smoking and risk of amyotrophic lateral sclerosis: a pooled analysis of 5 prospective cohorts. Arch Neurol 68, 207-213. 